\title{
Considerations for Use of Am-241 for Heat Source Material for Radioisotope Power Systems.
}

Stephen G. Johnson

January 2017

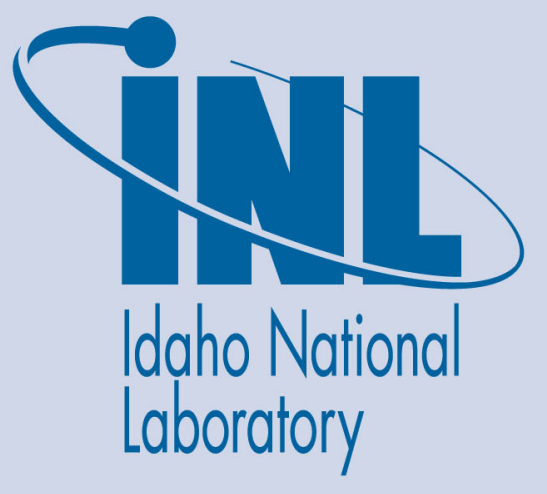

The INL is a U.S. Department of Energy National Laboratory operated by Battelle Energy Alliance 


\title{
Considerations for Use of Am-241 for Heat Source Material for Radioisotope Power Systems.
}

\author{
Stephen G. Johnson
}

January 2017

Idaho National Laboratory Idaho Falls, Idaho 83415

http://www.inl.gov

Prepared for the

U.S. Department of Energy

Under DOE Idaho Operations Office

Contract DE-AC07-05ID14517 


\section{Considerations for Use of Am-241 for Heat Source Material for Radioisotope Power Systems}

\section{Background}

Amercium-241 (Am-241) is an actinide created from irradiation of uranium in a nuclear reactor. It exhibits a 432-year half-life and decays primarily through alpha decay ( 5.44 and $5.48 \mathrm{MeV}$ ) with some relatively weak gamma rays (13.9 and 59.5 $\mathrm{keV}$ ). It does not directly emit either neutrons or beta particles although its daughter products may. It is primarily harvested from stockpiles of aged plutonium via decay of $\mathrm{Pu}-241$ (14.4 year half-life) and emission of a beta particle [1]. Typical amounts of Pu-241 in either reactor or weapons grade plutonium can be up to several percent. Plutonium recovered from the reprocessed Magnox reactor fuel in the United Kingdom can be as high as $11 \% \mathrm{Pu}-241$.

Industrial uses of Am-241 lie in principally 3 areas: 1) medical diagnostic analysis, 2 ) industrial neutron sources such as AmBe for use in measuring density and thickness and 3) smoke detectors.

Am-241 is controlled as "other accountable nuclear material" in the US under DOE order 474 .

\section{Use for Radioisotope Power Systems}

There have been several efforts made over the last 60 years to examine the entire periodic table of the elements to determine the best isotope/element for use as a heat source for radioisotope power systems (RPS). Am-241 is typically not in the final group being considered due to its 432-year half-life. The window of isotopes considered has usually been kept to 15-100 year half-life. The criteria in down selecting an isotope for use as a heat source material have been: 1 ) radiation characteristics (easy to shield), 2) specific power (higher is better), 3) fuel form considerations (chemically compatible with cladding, chemically inert with respect to the environment, robust mechanically, high melting point), and 4) available at a reasonable cost and suitable quantities.

There have been a few efforts examining Am-241 for use in RPS during the past 2 decades [2-4]. None of these has advanced beyond comparison of known chemical or material properties. No heat sources have been made and none have been placed into RPS for testing. No tests have been conducted of the various americium oxides ( $\mathrm{AmO}, \mathrm{Am}_{2} \mathrm{O}_{3}, \mathrm{AmO}_{2}$ nor nonstoichiometric phases between these oxides) for their behavior with the environment to confirm inert behavior or compatibility with cladding materials. From a crystallographic point of view americium and oxygen can form crystal phases that exhibit face-centered cubic, monoclinic, cubic or tetragonal characteristics. A simple point to make here is that the chemistry of 
americium and oxygen is complicated and not well understood at this point compared to plutonium and oxygen.

\section{Current or Possible Sources for Am-241}

There are two general sources of Am-241, domestic or foreign. There has been an effort underway to renew production of Am-241 at Los Alamos National Laboratory. This started in 2011 and was to be complete in 2015 but has suffered several delays. Current estimates have the project re-starting in early 2017 with production in earnest starting later in 2017. The project was delayed due to the work slowdown at TA-55 starting in 2015. The current campaign planned is to produce a few kilograms of Am-241 in 2017-2023. Predicted customer base for this production is thought to be primarily the drilling industry for AmBe sources. On a thermal basis a 5 kilogram amount of $\mathrm{Am}-241$ is equivalent to 1 kilogram of $\mathrm{Pu}-238$ isotope or $\sim 1.4$ $\mathrm{kg}$ of plutonium oxide production of $86 \%$ assay such as Oak Ridge National Laboratory intends to produce.

There was a proposal to separate $\mathrm{Am}-241$ from material $\left(\mathrm{PuO}_{2} / \mathrm{AmO}_{2}, 9: 1\right)$ sent to Savannah River Site (SRS) from Hanford [5]. If this was undertaken there is $\sim 17 \mathrm{~kg}$ of Am-241 present in this material. This was initial material for testing as a mixed oxide fuel for reactors. This effort was proposed but never initiated and its fate is likely to be tied to the mixed oxide fuel plant at SRS, which appears headed to the "cancelled project status" at present. No record was found of any other separated stores of Am-241 at the Hanford site.

The most relevant foreign source for RPS is likely to be the Sellafield plant in the United Kingdom (UK). The UK reprocessed Magnox fuel there for several decades and have a civilian stockpile of 100 MT of plutonium that was felt to easily yield at least 1 MT of Am-241. They are currently funded by the European Space Agency (ESA) to establish the separation techniques and facilities for full-scale operation. They began their efforts in 2011 [6]. The total scope for the UK includes: isotope separation, encapsulation, Radioisotope Thermoelectric Generator (RTG) development and Stirling Radioisotope Generator (SRG) development. Other aspects of the ESA effort were parceled out to other members of ESA. There is no current central technical integration function for this effort.

The UK effort also serves a secondary function or primary function depending on your viewpoint. The UK intends to produce mixed oxide fuel (MOX) for civilian nuclear reactors containing both uranium and plutonium oxide. The current planned MOX fuel manufacturing plant at Sellafield does not have extensive shielding for gamma radiation. The use of the Sellafield plutonium, which is planned, is likely to have too much Am-241 in it for safe use in the MOX plant. So the Am-241 must be removed or the design of the plant changed.

Below is an update on the current status of the ESA-funded UK effort from a visit with the Sellafield staff the week of October 3, 2016: 
National Nuclear Laboratory (NNL) personnel at their Sellafield location initiated $\sim 3+$ years ago R\&D work on the separation of Am-241 from spent nuclear fuel stocks being stored at their facility. It is estimated that the amount of Am-241 potentially available is in the range of greater than 1 MT and they are employing an oxalate process to separate the Am-241 from the spent fuel. Recent low-level experiments have proven successful as they have recovered low gram quantities of Am-241 with a very high purity of greater than $98+\%$. Current endeavors have been utilizing an in-house designed $500 \mathrm{ml}$ chemical reactor with the stated near term goal ( $\sim 2$ years out) of increasing output via the application of a 2 liter chemical reactor. The effort at NNL is being sponsored by a current contract from the European Space Agency. However, this contract is set to expire by the end of this calendar year and the follow-up contract may not be in place for $6+$ months. It is likely that there will be a hard stop in the current Am-241 activities at NNL until ESA funding resumes.

To date the only apparent work which has been performed on the sintering of NNL produced Am-241 oxide into ceramic pellets, occurred within the last year at Institute of Transuranium Elements (ITU) near Karlsruhe, Germany. Several very small pellets ( $\sim 4 \mathrm{~mm}$ in diameter $\mathrm{x} \sim 1 \mathrm{~mm}$ thick) were cold pressed and sintered. To our knowledge all of the very small pellets exhibited significant circumference cracking. It should be noted that ITU is a European Commission facility and not an ESA laboratory, so it is unlikely that full-scale Am-241 oxide pellets will be fabricated there for use in an RPS.

For the production of an Am-241 RPS system it is ultimately necessary to fabricate ceramic pellets of as yet undefined geometry and oxygen stoichiometry. It is anticipated that the fuel pellets will ultimately be "hockey puck" in geometry with a diameter of $\sim 5-8 \mathrm{~cm}$ and a thickness of $\sim 2-3 \mathrm{~cm}$. The Am/0 phase diagram is fairly complex, which will likely make pellet fabrication more difficult compared to ${ }^{238} \mathrm{PuO}_{2}$ pellet production. At the present time the fuel may be $\mathrm{Am}_{2} \mathrm{O}_{3}$, since it is perceived easier to fabricate into robust ceramic pellets compared to $\mathrm{AmO}_{2}$. There is general uncertainty in the ceramic processing approach, and it is likely that fuel fabrication may ultimately become a significant hurdle in the production of an Am241 fueled RPS.

The fielding of a "flight RPS" is thought to be doable in the middle to later part of the next decade (2024-30) if the various material issues are addressed successfully.

\section{Working with Am-241 versus Pu-238 for RPS Use}

US heat source production (Pu-238) currently takes place at Los Alamos National Laboratory in a different part of the same facility that will house the Am-241 production capability. The various gloveboxes are equipped with shielding specific to $\mathrm{Pu}-238$, primarily Lexan. These would have to be evaluated as to the amount of retrofitting or replacement that would be required to use Am-241 as heat source 
stock material. In recent years, the replacement of gloveboxes in TA-55 has been at a cost of $>\$ 5 \mathrm{M}$ per unit. The total number of gloveboxes is likely to be at least 10 . The changes to the safety authorization basis for TA-55 to accommodate heat source production would likely be costly and take considerable time. The UK effort has yet to devise suitable processing parameters to produce quality ceramic pellets for encapsulation thus making estimates on what changes to existing safety basis documents in the US facilities harder to estimate.

The current fueling and performance testing of the RPSs is conducted at the Idaho National Laboratory (INL). The fueling gloveboxes, and all shielding used in conjunction with the test equipment (vibration test apparatus, magnetic testing apparatus, center-of-mass instrument and thermal vacuum chambers) is specific to providing neutron shielding, which is constructed of light elements. In the case of the large fueling glovebox (Inert Atmosphere Assembly Chamber) its shielding is both lexan and water (12 inch thick water-filled windows and various shielding tanks). The use of a primary gamma-emitter such as Am-241 would require the use of leaded windows to ensure personnel safety. The quantity of Am-241 to be used for a Mars multi-mission RTG would be $\sim 17 \mathrm{~kg}$ of Am-241 isotope. While there would be some self-shielding at the heat source level and also shielding by the RPS shell itself the anticipated field would still be 100-300 mrem/hr gamma at a foot.

\section{Other Facilities to be Considered for Am-241 Effort}

There has not been use of large quantities of Am-241 in the US in the past. For example, although there are many smoke detectors produced domestically each contains less than 1 microcurie of Am-241, i.e., less than 1 microgram of Am-241 [ 1 gram of Am-241 will make roughly 3 million smoke detectors]. Other gamma radiation emitters are usually handled in hot cells or similar types of shielded facilities. There are several operational hot cells in the US but these facilities are not conducive to the delicate operations involved in fueling and testing of RPS and are either already fully subscribed or are in need of extensive and expensive repairs.

\section{Use of a Flight-ready and Fully Fueled Am-241 RPS Supplied by ESA}

The various challenges yet to be overcome for the ESA-led effort have been delineated above. The use of an ESA-supplied RPS for launch from Kennedy Space Center involves some additional challenges. One being that for RPS of size similar to the MMRTG powering the rover Curiosity ( 110 We at beginning of mission) transportation is done using a Type B shipping container which is actively cooled during transport. This cooling preserves the thermoelectrics from degradation that can occur at higher temperatures. If the RPS uses another type of conversion system, such as a Stirling engine, the rare earth magnets need to be cooled to prevent de-naturing. There is only one such Type B shipping container (9904 RTG Shipping Container) and it is qualified for international shipments but is only certified for $\mathrm{Pu}-238$ payloads at present. This container would need to be recertified for an Am-241 payload which may involve additional lead shielding to meet 
the Department of Transportation specifications for radiation field outside the transportation trailer.

Another challenge would be ensuring that the safety testing performed by ESA was answering the needs of launch approval for a US launch. Any delta in the data supplied by ESA and needed by the US would need to be addressed.

\section{Brief Comparison of Am-241 vs. Pu-238 Fueled Systems based on Historical Data}

The GPHS-RTG that flew to Pluto recently weighed 127 lbs fully fueled with the weight of the 18 GPHS step 1 modules with Pu-238 fuel constituting 60 lbs of that total weight. The MMRTG that powers Curiosity on Mars weighs 100 lbs fully fueled with the weight of 8 GPHS step 2 modules constituting $\sim 28 \mathrm{lbs}$ of that weight. The lower specific power of Am-241 and subsequent greater mass would increase the weight of the fuel for these two convertors as follows: GPHS-RTG from $60 \mathrm{lbs}$ to 300 lbs and for MMRTG from $28 \mathrm{lbs}$ to $140 \mathrm{lbs}$. These calculations assume that as the heat source increases in geometrical size, as it must, that the amount of specialized carbon-carbon material encapsulating it must scale accordingly. Some change to the weight of the convertors would also occur although that change will not be speculated on here. These changes in weight could have significant consequences to NASA missions that tend to be very weight conscious.

\section{Summary}

The following statements are the concluding remarks of this study:

1) The US lacks sufficient domestic production capabilities for a viable Am-241 RPS program.

2) The existing infrastructure for Pu-238 RPS is likely to require extensive and expensive retrofitting to handle a gamma emitter such as Am-241 versus the existing neutron emitter-Pu-238. The cost of completely new US facilities would be equally expensive or more so.

3) The ESA-led effort is at least 10-12 years away from providing either Am-241 raw material or Am-241 fueled RPS. There are substantial risks in establishing the use of Am-241 as a heat source. These include unknown chemical behavior of Americium oxide with the environment, i.e., such as in a launch accident scenario and material properties conducive to manufacturing a cohesive ceramic heat source with robust mechanical properties.

4) It is unclear to what extent the launch safety testing for the ESA-led program for the Am-241 RPS would "map" one-for-one to the current US requirements. This could lead to additional schedule being added onto the 10-12 years mentioned above if the ESA testing/analysis needs to be supplemented. 
5) The 10-12 years mentioned above is a best case scenario and assumes that the US needs are first in line before ESA needs which may be an unrealistic assumption. Also the NEPA action for a nuclear-enabled launch in the US typically starts 7 years before launch. The design of the heat sources and/or the power conversion system would have to be fairly well defined to initiate the NEPA process. This is likely to add additional time onto the 10-12 years mentioned above.

\section{$\underline{\text { References }}$}

[1] Gray, L. W. et al., "Recovery of Americium-241 From Aged Plutonium Metal”. DP1577, December 1980, SRL.

[2] Robinson, G. A., et al., "Preliminary Analysis: Am-241 RHU/TEG Electric Power Source for Nanosatellites", Nuclear and Emerging Technologies for Space Applications, February 2014.

[3] O’Brien, R. C., et al., "Safe Radioisotope Thermoelectric Generators and Heat Sources for Space Applications”, J. Nucl. Mat. 377, pp 506-521 (2008).

[4] Schmitz, P. C. and Penswick, L. B., "Lunar Surface Stirling Power Systems Using Am-241", 7th Int. Energy Conversion and Engineering Conf., August 2-5, 2009, Denver CO.

[5]Lessing, P. A. and Glagolenko, I., "High Purity Amercium-241 for Fuel Cycle R\&D Program", Inst. Nucl. Mater. Mang. 2011, Palm Springs, CA, July 17-21, 2011.

[6] Rice, T., "Space Nuclear Power Systems. Activities and Programmes in the United Kingdom", Committee on the Peaceful Uses of Outer Space Scientific and Technical Subcommittee, 51st session, Vienna, February 10-21 2014. 